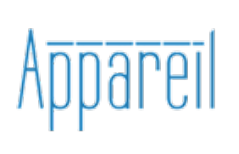

Appareil

$6 \mid 2010$

Philosophie et cinéma

\title{
D'un détournement mineur du discours médical sur le sexe (Glen or Glenda, 1953)
}

Alain Naze

\section{(2) OpenEdition}

1 Journals

\section{Édition électronique}

URL : http://journals.openedition.org/appareil/1082

DOI : 10.4000/appareil.1082

ISSN : 2101-0714

Éditeur

MSH Paris Nord

Référence électronique

Alain Naze, «D'un détournement mineur du discours médical sur le sexe (Glen or Glenda, 1953) », Appareil [En ligne], 6 | 2010, mis en ligne le 07 octobre 2010, consulté le 30 juillet 2020. URL : http:// journals.openedition.org/appareil/1082 ; DOI : https://doi.org/10.4000/appareil.1082

Ce document a été généré automatiquement le 30 juillet 2020.

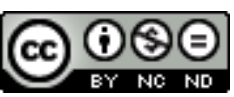

Appareil est mis à disposition selon les termes de la Licence Creative Commons Attribution - Pas d'Utilisation Commerciale - Pas de Modification 4.0 International. 


\title{
D'un détournement mineur du discours médical sur le sexe (Glen or Glenda, 1953)
}

\author{
Alain Naze
}

1 Le film que je vais évoquer est Glen or Glenda, d'Ed Wood, lui-même surnommé « le pire réalisateur de tous les temps", comme le rappelle le film que Tim Burton lui a consacré. Ce film de 1953 relève de ce qu'on a appelé aux États-Unis le «cinéma d'exploitation », c'est-à-dire d'un cinéma en marge des circuits de production et de diffusion officiels. Ce cinéma parallèle échappait en fait aux multiples formes d'interdiction propres au code Hays (qui s'est appliqué, plus ou moins strictement, de 1934 à 1966). Ayant émergé au lendemain de la première guerre mondiale, ce « cinéma d'exploitation », dépourvu de moyens, se maintiendra malgré tout jusque dans les années 1960, 1970 - les films qui en seront issus prendront notamment en charge les cinémas dits "ethniques", ou encore les thèmes interdits dans les productions traditionnelles. Si, par ce moyen, certaines thématiques ont pu en effet être traitées en échappant aux multiples formes de censure du code Hays, il ne faudrait pourtant pas se faire du « cinéma d'exploitation » une image militante, comme le souligne Didier RothBettoni, dans son livre L'homosexualité au cinéma :

[...] rien n'était tabou pour le cinéma d'exploitation, l'homosexualité pas plus qu'un autre sujet dans la mesure où cela pouvait titiller un certain public en mal de sensations. Il ne faut en effet jamais perdre de vue que le ciné d'exploitation, aussi libératoire a-t-il pu être, a toujours été le contraire d'un cinéma militant: sensationnalisme et voyeurisme sont en effet systématiquement au programme, la plupart du temps sous de pseudo prétextes scientifiques, et le progressisme est loin d'être de rigueur, ces débordements finissant toujours par rentrer dans le rang grâce à un discours moral sous-jacent tranchant hypocritement avec ce qui s'est déroulé sur l'écran'.

2 En ce qui concerne le film d'Ed Wood, on verra si, au bout du compte, en effet, tout finit par rentrer dans le rang, mais faisons d'abord remarquer qu'après la seconde guerre mondiale, aux États-Unis, la question des déviances sexuelles quitte largement le registre moral et social (la déviance sexuelle comme perversion morale et comme fléau 
social) pour investir celui de la pathologie, et passer ainsi dans le domaine des questions médicales. Ce déplacement constitue également un des enjeux de Glen or Glenda, d'un point de vue stratégique, et la question qu'on devra se poser est celle des limites d'un tel déplacement : si le travestisme et la transsexualité gagnent un droit de cité à travers ce changement de registre, le prix à payer n'est-il pas trop lourd ? On n'oubliera pas au passage que c'est surtout la question du travestisme qui intéressait le réalisateur (se livrant ici à un plaidoyer pro domo), la question de la transsexualité s'étant ici trouvée abordée en même temps, sans doute surtout du fait que l'affaire Christine Jorgensen (celle du premier américain qui, en 1952, avait changé de sexe) avait eu un grand retentissement dans l'opinion publique.

Dès le préambule, et malgré la présence d'un narrateur, incarné par Bela Lugosi en l'occurrence, Glen or Glenda veut se donner à voir, essentiellement, comme un documentaire, avec une intention positiviste affichée : «Ce film est réaliste et refuse de prendre parti. Seuls les faits sont montrés, tels qu'ils sont. Vous êtes la société, NE JUGEZ PAS ». La visée essentielle, consistant à se défaire de tout jugement moral en ces questions de sexualité, est évidente, et c'est dans cette optique que l'usage que fera ici Ed Wood du discours médical peut être envisagé comme une forme de détournement: mutatis mutandis, cette démarche s'apparente à celle de Magnus Hirschfeld, opérant tout un déplacement du discours sur le sexe vers des catégories médicales. Pour l'homosexualité, il est évident que bien des invertis (selon la dénomination qui précéda l'adoption du terme d'homosexuel) étaient intéressés par la constitution d'une subjectivité homosexuelle, notamment en ce qu'une reconnaissance médicale de la possibilité de tendances sexuelles non hétérosexuelles ouvrait éventuellement la voie à un traitement possible, ou du moins à un accompagnement médical, en lieu et place d'une pure et simple répression. Ed Wood jugeait lui-même comme un progrès (à la fois de la raison, de la technique et des mœurs) une vision médicale du travestisme et de la transsexualité, si l'on en juge par le parallèle qu'il effectue entre le fait, au milieu du $\mathrm{xx}^{\mathrm{e}}$ siècle, d'être choqué par un changement de sexe chirurgical, et le fait, dans des époques antérieures, d'avoir été choqué de ce que l'homme avait voulu voler dans les airs, ou se déplacer plus rapidement en roulant en voiture; il considère en cela que la rationalité médicale combat les préjugés du type : «Si le créateur avait voulu que nous volions, il nous aurait donné des ailes ", ou plus précisément, à travers une voix féminine : «Si le créateur avait voulu que nous soyons des garçons, nous serions nées garçons ». Ed Wood considèrera donc que la nature commet des erreurs, qu'il s'agit de rectifier si possible; lorsqu'apparaît l'image d'un homme travesti en femme, le commentaire énonce ceci : " La nature commet des erreurs, chaque jour en apporte la preuve. Cette personne est un travesti. Un homme qui se sent mieux dans des vêtements de femme. "Travesti" est le terme scientifique pour désigner ceux qui s'habillent comme le sexe opposé. Beaucoup de travestis veulent être du sexe opposé ». On utilise ici, d'emblée, des catégories dites "scientifiques", afin de rationaliser le rapport qu'on peut entretenir vis-à-vis du fait de se travestir, ou de changer de sexe, quitte à recourir aussi à l'argument de l'utilité sociale pour justifier le travestissement : "Avec des dessous de satin, une robe et un pull moulant, ou un pyjama féminin, cet homme est le plus heureux du monde. Il travaille et réfléchit mieux. Il sert mieux sa communauté et son gouvernement, parce qu'il est heureux ». En n'hésitant pas à endosser l'étiquette que la science médicale attribue à certains comportements et/ou identités sexuels, des individus stigmatisés peuvent en effet tenter d'entrer dans un tel processus d'assujettissement, stratégiquement, notamment pour écarter certaines 
formes de répression; c'est ce que souligne Michel Foucault: «La résistance prend toujours appui, en réalité, sur la situation qu'elle combat. Dans le mouvement homosexuel, par exemple, la définition médicale de l'homosexualité a constitué un outil très important pour combattre l'oppression dont était victime l'homosexualité à la fin $d u x x^{e}$ siècle et au début $d u x^{e}$. Cette médicalisation, qui était un moyen d'oppression, a toujours été aussi un instrument de résistance, puisque les gens pouvaient dire: "Si nous sommes malades, alors pourquoi nous condamnez-vous, pourquoi nous méprisez-vous? ", etc. Bien sûr, ce discours nous paraît aujourd'hui assez naïf, mais, à l'époque, il était très important ${ }^{2}$ ». Face à la menace répressive, la définition médicale a pu alors évidemment paraitre libératrice, et c'est encore aujourd'hui, dans certaines parties du monde, l'angle d'attaque que peuvent privilégier certaines minorités sexuelles, craignant pour leur vie, précisément pour ces raisons de sexualité. Cette dimension tragique n'est pas absente de Glen or Glenda non plus, une des premières scènes du film étant celle du suicide d'un travesti.

4 À sa forme "documentaire ", le film va adjoindre le récit de «l'étrange cas de Glen et Glenda, une seule et même personne ", non pas "mi-homme mi-femme", mais " homme et femme dans un même corps, même si son apparence extérieure est celle d'un homme ». On voit déjà que la distinction des sexes vise ici à être maintenue, en ce que la définition de ce travesti comme «mi-homme mi-femme » est écartée et, dans le même ordre d'idée, c'est l'indistinction avec l'homosexualité qui est combattue, la voix du commentaire précisant en effet: "Glen est un travesti, pas un homosexuel. Travestisme est le terme médical pour désigner ceux qui s'habillent comme l'autre sexe et dont la sexualité est tout à fait normale ». On aperçoit immédiatement les limites de ce type de stratégie: pour gagner en respectabilité, on cherche à se distinguer de certaines autres catégories stigmatisées, que l'on confirme alors dans leurs stigmates. Glen serait d'ailleurs si peu suspect d'homosexualité qu'il est sur le point d'épouser Barbara, le seul problème étant que cette dernière ignore encore la double personnalité de Glen, et toute une partie du film va tourner autour de l'aveu que le futur marié doit faire à sa fiancée. Celle-ci, d'ailleurs, semble si loin de ces questions que l'évocation, dans le journal, d'un homme ayant changé de sexe, amène sur ses lèvres les mots suivants :

Je me demande ce que je ferais à leur place, si j'avais l'esprit aussi troublé qu'eux, ou si je réalisais que ça ne tourne pas rond chez toi. C'est dur à imaginer. Nous, un couple normal, sur le point de se marier et de vivre normalement. Et ce malheureux, qui n'aurait jamais trouvé le bonheur sans la médecine.

5 Que le couple soit jugé «normal » écarte encore le soupçon d'homosexualité ou de sexuation flottante chez Glen, mais en même temps, en unissant ici bonheur et médecine, Barbara anticipe sur la fin du film, les deux destins heureux (d'Alan/Anne et de Glen/ Glenda) en passant par un accompagnement médical, voire chirurgical. Or, si la médecine et ses techniques peuvent rectifier ce que la nature a mal fait, c'est donc qu'elle ne s'oppose en rien à la nature elle-même - et l'on comprend déjà que les catégories écartées n'auront pas la même « chance ", et c'est là que résident les « effets de misère » qui handicapent la démarche d'ensemble du film. «Glen devrait consulter un psychiatre » indique le commentaire, en guise de conseil pour un bonheur à venir : "Glen/Glenda doit prendre une décision, ou renoncer à épouser Barbara ». Et c'est le passage à l'aveu qui va ouvrir la voie à tout un discours pseudo-psychiatrique, pseudoanalytique, parfois proche des raccourcis théoriques qu'il arrive à Hitchcock d'emprunter, lorsqu'il fraie dans ces parages. 
6 Lorsque la scène de l'aveu a lieu, d'abord désarçonnée, Barbara devient pourtant rapidement compréhensive: «Glen, je ne comprends pas tout, mais nous trouverons une solution ensemble ", au point qu'elle lui donne le pull angora qu'il dévorait des yeux et caressait, fasciné. Cette solution qu'ils trouveront ensemble, c'est celle qui émergera de la fréquentation d'un psychiatre par Glen, puis par le couple réuni, dans une scène chez ce psychiatre, l'une des dernières du film. Mais avant qu'intervienne cette scène, le psychiatre racontant à son interlocuteur (sans doute un journaliste) l'histoire de Glen, tient à la placer en résonance avec une autre histoire, celle d'Alan, qui deviendra Anne - ainsi, au cas de Glen/Glenda, jugé « l'un des plus simples », vient s'ajouter un cas de transsexualité. Si, donc, tous deux se travestissent, l'un serait " malheureux» s'il changeait de sexe (Glen), l'autre serait "malheureux » s'il ne changeait pas de sexe (Alan). La distinction sur laquelle va alors s'appuyer le psychiatre renvoie d'un côté à une question strictement mentale (pour le cas de Glen, il indique en effet que «[1]e problème est dans la tête et remonte à son enfance »), et de l'autre côté à une question à la fois mentale et physique (concernant Alan, pour l'aspect mental, il est indiqué ceci : «La mère d'Alan voulait une fille. À son père, ça lui était égal. Alan n'aimait pas les sports dont tous les garçons raffolent. [...] Il s'intéressait aux sports féminins. [...] Il aimait les travaux ménagers. Alan devenait une femme. Dans sa tête seulement, mais la tête gouverne "; quant à l'aspect physique, sur lequel va pouvoir s'appuyer le discours de légitimation de l'opération de changement de sexe, comme rectification d'une « erreur de la nature », le psychiatre considère qu'Alan entre dans la catégorie des " pseudo-hermaphrodites »: «Il fut largement examiné par d'éminents médecins et moi-même. Alan était un pseudo-hermaphrodite. Un hermaphrodite possède les deux organes sexuels parfaitement formés. Chez le pseudo-hermaphrodite, l'un des deux organes est bien formé, l'autre est imparfait et difficile à déceler. Alan fait partie de ces derniers. Alan voulait choisir. La nature s'était trompée. Il nous demanda de corriger cette erreur. Alan devait choisir entre être un homme ou devenir une femme. Les deux étaient possibles »). Tout comme le fait d'indiquer que Glen avait une sexualité "normale ", c'est-à-dire hétérosexuelle, empêchait le film d'ouvrir sur une prise en compte tolérante de l'homosexualité (qui semblait alors reléguée dans les anomalies contre-nature), de façon à déconnecter très nettement le travestisme et l'orientation sexuelle, de la même manière, donc, le fait de relier le travestisme d'Alan au fait qu'il serait "pseudo-hermaphrodite" empêche d'ouvrir une zone d'indiscernabilité entre les sexes. En effet, selon le propos du film, Alan était physiquement susceptible d'être homme ou femme, et donc l'opération de changement de sexe ne vise qu'à clarifier la situation : il doit choisir entre être un homme ou une femme - il s'est comporté comme un homme, au combat, et après l'opération il aura à apprendre comment se comporte en toutes choses une femme. La différence des sexes est donc clairement réaffirmée, et en cela, le travestisme est donc aussi coupé de ses potentialités de déterritorialisation à cet égard : chacun de nous aurait bien un sexe, qui serait le bon, et les opérations de changement de sexe ne se justifieraient donc que lorsque la nature a commis une erreur, en restant dans l'indétermination - l'opération de changement de sexe ne viserait, par conséquent, qu'à rétablir un ordre qui serait celui de la nature elle-même, l'ordre de la différence des sexes, au sein de laquelle seuls deux sexes, opposés, existent. On voit bien ici l'usage qui est fait, par Ed Wood, du discours médical ou pseudo-scientifique sur le sexe : il s'agit de s'appuyer sur cette autorité (incarnée par le personnage du psychiatre) pour légitimer certaines pratiques, ou certaines transformations physiques. Et l'on retrouve le lien entre médecine et 
bonheur dans ces mots du psychiatre : «Enfin, Alan est Anne, une jeune fille épanouie, fruit de la médecine moderne, comme le monstre de Frankenstein. [...] elle était faite pour être une femme. Depuis l'opération, elle est une femme heureuse ", en même temps que l'aspect normatif du pouvoir médical: «Continuant mon travail de psychiatre, j'explique à Anne les devoirs d'une femme ».

7 Le cas de Glen, lui, est très différent, selon les mots du même psychiatre, qui se fait d'ailleurs psychanalyste ici : "Glen n'a qu'un organe sexuel : le mâle. Au cours de mes séances, j'ai appris plusieurs choses. Le père de Glen ne l'aimait pas. [...] Sa mère ellemême avait détesté son propre père. Glen lui rappelait ce père. Elle reporta alors tout son amour à sa fille. Glen décida alors de devenir une fille ». Cette psychanalyse expéditive tend à indiquer que le problème de Glen n'ayant nulle origine physique, il peut faire disparaître son double féminin, s'il le désire. La compréhensive Barbara concède que si le personnage de Glenda ne disparaissait pas, cela ne serait pas si grave, et la fin, idyllique, est indiquée : « Le temps passe, et grâce à son mariage heureux, au traitement psychiatrique, à l'amour et à la compréhension de Barbara, Glen commence à oublier Glenda. Il a enfin une mère, une sœur, une épouse, et Glenda en une seule et adorable personne. Le cas de Glen finit donc bien ». On remarquera que le réalisateur est au bout du compte contraint d'accepter l'idée d'une sorte de guérison possible, ce qui est le prix à payer pour cette prise en charge médicale et compréhensive du travestisme et de certains cas de changement de sexe. Même si l'intention d'Ed Wood est de rendre plus vivable la vie des travestis, la logique du discours qu'il utilise ici de façon stratégique est pourtant de conduire à une éradication tendancielle de ce type de comportement. L'arme utilisée est donc bien à double tranchant, et si elle provoque par ailleurs des "effets de misère ", on peut finalement se demander s'il n'est pas, d'un certain point de vue, préférable d'échapper à une telle prise en charge par le discours médical, même à des fins stratégiques.

On ne peut en effet, en voyant Glen or Glenda, se défendre contre l'impression déplaisante selon laquelle le réalisateur serait prêt à sacrifier quelques victimes "collatérales" au profit de la cause qu'il défend - celle du travestisme, et accessoirement de certaines formes de transsexualité. Au point qu'on en arrive à se dire que ce film aurait un effet de libération plus marqué s'il n'excluait pas l'homosexualité, ou les opérations de changement de sexe non justifiées par la présence de deux organes sexuels, mâle et femelle, etc. Or, ce faisant, on tend à adopter le point de vue selon lequel une telle prise en charge du sexe par le discours médical et scientifique est souhaitable, du point de vue d'une libération. Pourtant, on a bien lu Foucault, et on connait bien les effets indésirables de cette stratégie, mais tout se passe comme si, à travers ce retour dans les années cinquante du code Hays, on retombait dans l'illusion selon laquelle parler de sexualité, quelle que soit la manière dont on en parle, revient à libérer le sexe lui-même. Et l'on serait presque prêt à argumenter, pour montrer en quoi l'homosexualité, par exemple, n'est pas plus contre-nature que le travestisme, c'est-à-dire qu'on serait au fond presque prêt à prendre parti dans un discours pseudo-médical sur le sexe. Il est donc une fois de plus utile de revenir à la Volonté de savoir (Michel Foucault), qui nous montrait bien comment c'est tout un ensemble de catégories, de l'homosexuel à l'androgyne en passant par le masturbateur, ou encore par les enfants précoces ou les pédophiles, qui prennent naissance dans le sillage de la prolifération des discours sur le sexe : à la jonction des préoccupations du médecin, du psychiatre, du juge, du policier, naît donc ce peuple apparenté aux monstres (on pourrait penser au personnage central de M Le maudit, de Fritz Lang), que 
l'on caractérise comme pervers, à la frontière de la délinquance et de la folie, dans les classifications institutionnelles. Car alors, il s'agit de bien saisir, et de ne pas oublier, que ce n'est pas d'abord, que ce n'est pas essentiellement une logique d'exclusion qui est à l'œuvre dans ce type d'entreprises de classification systématiques dans le domaine sexuel : il ne s'agit pas d'exclure "mille sexualités aberrantes", mais de recourir à une "spécification, [à une] solidification régionale de chacune d'elles ", c'est-à-dire, au fond, qu'il s'agit, ainsi, "en les disséminant, de les parsemer dans le réel et de les incorporer à l'individu ${ }^{3}$ ». Cette manière de définir l'objet "sexualité » permet de penser le basculement entre un pouvoir de type souverain, essentiellement répressif, et un biopouvoir, de type essentiellement pastoral. C'est à partir de là qu'il y a lieu d'être méfiant à l'égard de l'idée d'une libération sexuelle entendue comme émancipation sexuelle intimement liée à une libération/prolifération des discours sur le sexe. Là où le travesti, le transsexuel ou l'homosexuel peuvent opérer leur coming out ou son équivalent, ils ne peuvent d'abord le faire que dans les termes d'une identité contrainte, c'est-à-dire reçue à partir des techniques classificatoires de la médecine et de la psychiatrie - on comprend d'ailleurs, dans cette optique, que toute une partie de Glen or Glenda se déploie dans le registre de l'aveu, premier pas vers la rédemption promise par la médecine et l'amour réunis.

\section{BIBLIOGRAPHIE}

Foucault Michel, La volonté de savoir, Paris, Gallimard, 1976

Foucault Michel, Dits et écrits II, 1976-1988, Paris, Gallimard, 2001.

Roth-Bettoni Didier, L'homosexualité au cinéma, Paris, La Musardine, 2007.

\section{NOTES}

1. Didier Roth-Bettoni, L'homosexualité au cinéma, Paris, La Musardine, 2007, p. 126.

2. Michel Foucault, Dits et écrits II, 1976-1988, Paris, Gallimard, 2001, p. 1560.

3. Michel Foucault, La volonté de savoir, Paris, Gallimard, 1976, p. 60.

\section{RÉSUMÉS}

Relevant du " cinéma d'exploitation », si Glen or Glenda est encore capable de retenir l'attention du spectateur aujourd'hui, c'est sans doute moins grâce aux qualités intrinsèques du film luimême, qu'eu égard à la hardiesse du propos aux temps du code Hays - les thèmes qui y sont 
abordés vont en effet du travestisme à la transsexualité en passant par l'homosexualité. Il serait cependant exagéré de conclure au caractère uniment libérateur du film d'Ed Wood, ne serait-ce, par exemple, que du fait que le travestisme y est défendu largement au détriment de l'homosexualité.

Quoi qu'il en soit, l'intérêt majeur de cette réalisation réside sans doute dans le fait que l'aspect normatif du propos (la médecine envisagée comme un moyen de rectifier des erreurs commises par la nature), dominant les discours des années 1950, ne parvient pas à empêcher que lèvent ici les prémices du paradigme contemporain de la tolérance. En cela, ce film célèbre en quelque façon les noces inattendues de la médecine et de l'amour.

INDEX

Mots-clés : aveu, identité, médecine, tolérance, transsexualité, travestisme

Personnes citées : Wood (Ed)

\section{AUTEUR}

\section{ALAIN NAZE}

Professeur de philosophie à Quimper, Alain Naze est titulaire d'un doctorat en philosophie obtenu auprès de l'université de Paris 8 . Auteur de Temps, récit et transmission chez W. Benjamin et P.P. Pasolini aux éditions l'Harmattan. 